\title{
Consistent Indicators and Methods and a Scalable Sample Design to Meet Assessment, Inventory, and Monitoring Information Needs Across Scales
}

\author{
By Gordon R. Toevs, Jason W. Karl, Jason J. Taylor, \\ Carol S. Spurrier, Michael "Sherm" Karl, Matthew R. Bobo, \\ and Jeffrey E. Herrick
}

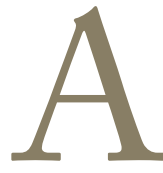

ccurate and timely information on the condition and trend of natural resources is crucial for making effective management decisions. Within the Bureau of Land Management (BLM), as in many land management agencies throughout the world, much effort is invested in monitoring for and assessing specific management needs. Because these specific management needs are often focused on a single objective, the data often are of limited utility for other management objectives. The ability to use monitoring data for more than one purpose is becoming increasingly important as the use of public lands simultaneously intensifies and diversifies. It is not efficient, nor ultimately effective, to develop individual monitoring plans for different management objectives (e.g., resource planning, permit compliance, restoration, recreation impacts, invasive species, and energy development). An assessment, inventory, and monitoring approach built on consistent indicators of ecological function and capacity (that can be supplemented with additional indicators for local needs or specific ecosystem characteristics) can provide data that can be combined to address multiple needs at multiple scales.

Many management issues faced by the BLM and other land management agencies are inherently multiscale in nature. Local decisions are made at the scale of a BLM field or district office; they require local monitoring and assessment information, and they can be informed by broad-scale information. Other decisions are made at broader scales (e.g., state or national levels) and need consistent information collected across management boundaries. An example of a multiscale management issue is the impact of invasive species. At a local level, the distribution and prevalence of invasive species can be assessed through individual field surveys, and appropriate management can be implemented and monitored over time. Consistent monitoring of invasive species across all field offices in a state is necessary to detect broad-scale patterns (e.g., linking invasive species distribution to land uses). Monitoring and assessment data collected to meet the local management needs should also contribute to regional and national monitoring and assessment efforts.

The BLM Assessment, Inventory, and Monitoring (AIM) Strategy was initiated, in part, to evaluate and to make recommendations for improving the efficiency and effectiveness of monitoring activities. ${ }^{1}$ A goal of the AIM Strategy is to provide the BLM and its partners with the information needed to understand terrestrial resource location and abundance, condition, and trend, and to provide a basis for effective adaptive management. The Strategy supports an integrated approach that includes three components:

1) A standard set of field-measurement indicators and associated methods for terrestrial vegetation and soils that reflect the status of key attributes of ecosystem sustainability

2) A statistically valid sampling framework that allows data sets collected in different areas and for different objectives to be aggregated at different scales to address regional and national information needs 


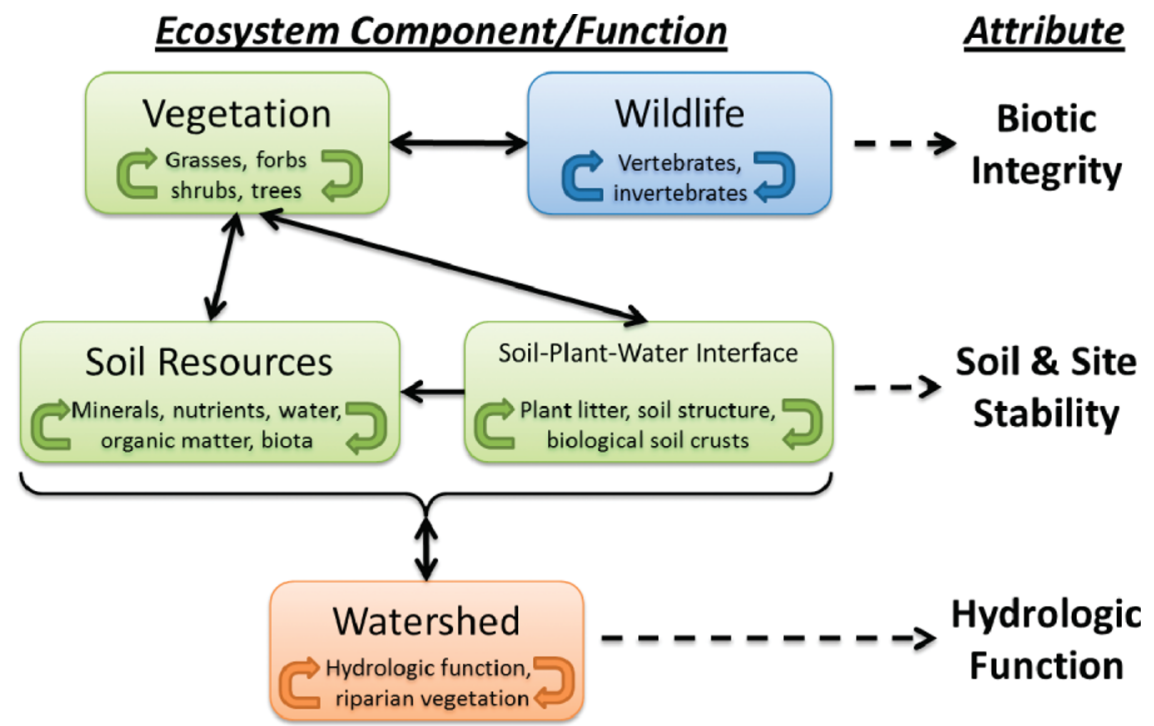

Figure 1. Assessing and monitoring natural systems requires consideration of the major structural components of ecosystems (boxes) and their functional relationships (solid arrows). Biotic integrity, soil and site stability, and hydrologic function have been identified as three key attributes that can be evaluated to determine ecosystem function., ${ }^{213}$ Figure inspired by Miller. ${ }^{14}$

3) Integration of remote sensing and ground-based technologies to maximize BLM's capacity to cost-effectively address management questions at multiple spatial scales

Collectively, these components help ensure that data collected to support local decision making are defensible and can be easily integrated to address multiple questions at multiple scales. Our objective in this paper is to provide an overview of these three components. We recognize that the BLM has a large amount of legacy data and data collection programs that do not adhere to these components yet continue to have value for supporting some management objectives. We describe a strategy to ensure that future data collection will have greater utility to address a broad range of objectives, and to the extent possible, will be statistically defensible. This strategy can also be used, as time and resources permit, to improve existing data collection efforts.

\section{Key Attributes of Ecosystem Sustainability}

All three components above are designed to increase the ability to detect changes in three key attributes of ecosystem sustainability on which virtually all land uses depend ${ }^{2}: 1$ ) soil and site stability, 2) hydrologic function, and 3) biotic integrity (Fig. 1). Soil and site stability refers to the capacity of a site to limit redistribution and loss of soil resources (e.g., nutrients and organic matter) by wind and water. Hydrologic function is the capacity of a site to capture, store, and safely release water from rainfall, run-off, and snowmelt. Biotic integrity is defined as the capacity of a site within an ecosystem to support natural processes within a normal, or expected, range of variability. Collectively, ecosystem status can be determined by comparing measured indicators of the three attributes to what is expected based on site potential. ${ }^{3}$
The approach we describe here also allows individual management objectives to be more directly addressed through 1) generation of additional indicators from the standard methods and 2) inclusion of supplementary methods.

\section{Core Indicators and Methods}

Sampling under the AIM Strategy is based on a suite of consistent indicators of the three key attributes of ecosystem sustainability (i.e., what to measure) and specific, repeatable, easy-to-implement methods for measuring those indicators (i.e., how to measure). The core indicators were developed by a collaborative process that synthesized knowledge and experiences of nearly 200 scientists, rangeland managers, and ecologists from different agencies and institutions. ${ }^{4}$ A large number of potential indicators were rated against 16 criteria, and the most general indicators with the broadest applicability to a range of management questions were selected. Standard methods for measuring the AIM Strategy indicators were selected that met the following criteria: 1) well documented, 2) widely used, 3) easy to implement, and 4) minimal potential for bias. An effort was made to identify methods that were used in existing monitoring programs and that could be used to measure multiple indicators. The extent to which comparable data can be collected using remote-sensing technologies was also considered. The core indicators and methods are also nationally applied to rangelands by the NRCS National Resources Inventory (NRI) program, ${ }^{5,6}$ which was extended in 2011 to BLM managed lands in the lower 48 states.

To interpret indicator measures for a single point in time or to establish a trend over time, the indicator values must be evaluated in relation to on-the-ground site characteristics 
Table 1. Core and contingent indicators for the Assessment, Inventory, and Monitoring Strategy and their recommended collection methods. Core indicators are always implemented. Contingent indicators are only implemented if there is reason to believe they are necessary for monitoring or assessing the site.

\begin{tabular}{|c|c|c|c|}
\hline Type & Indicator & Method* & Where applied? \\
\hline \multirow[t]{6}{*}{ Core } & Amount of bare ground & \multirow{4}{*}{$\begin{array}{l}\text { Line-point intercept (LPI, including } \\
\text { modifications described in Table } \\
\text { 2) supplemented with plot-level } \\
\text { species inventory }\end{array}$} & \multirow[t]{4}{*}{ All vegetation monitoring } \\
\hline & Vegetation composition & & \\
\hline & Non-native invasive species & & \\
\hline & $\begin{array}{l}\text { Plant species of management } \\
\text { concern }\end{array}$ & & \\
\hline & Vegetation height & Height at selected LPI points & All vegetation monitoring \\
\hline & $\begin{array}{l}\text { Proportion of site in large, } \\
\text { intercanopy gaps }\end{array}$ & Canopy gap intercept & All vegetation monitoring \\
\hline \multirow[t]{2}{*}{ Contingent } & Soil aggregate stability & Soil stability & $\begin{array}{l}\text { When soils are potentially } \\
\text { unstable (most rangeland) }\end{array}$ \\
\hline & Significant accumulation of toxins & Sampling for toxins in soil & $\begin{array}{l}\text { When toxins are believed present } \\
\text { (e.g., chemical spills) }\end{array}$ \\
\hline
\end{tabular}

developed for areas having similar potential to produce vegetation and response to management actions and disturbance. For instance, an increase in the amount of bare ground may indicate a positive or negative trend, depending on the site potential or management of the site. An increase in the amount of bare ground in a sagebrush (Artemisia spp.) plant community could signify excessive livestock grazing or drought. However, a sagebrush plant community at site potential may have greater bare ground when compared against cheatgrass (Bromus tectorum)-infested areas. Interpretation of indicators is facilitated by knowing their expected ranges for a site and by using multiple indicators (e.g., adding cover of invasive species to the above example) to provide observations against which changes can be compared. The AIM Strategy relies on ecological site descriptions developed by the NRCS for determining site potential and expected indicator values.

\section{Core Indicators}

The indicators and methods supported by the AIM Strategy (Table 1) represent a minimum set of information for new and, to the extent possible, existing monitoring projects. Core indicator data sets from different areas collected using the core methods can be combined at scales larger than an individual monitoring program. To meet local information needs, additional indicators can be calculated from the core methods and, where necessary, supplemented by additional methods.

The AIM Strategy recommends that six core indicators be quantified wherever BLM implements quantitative vegetation or soil monitoring in grassland, savanna, woodland, forest, and riparian ecosystems. Bare ground is one of the most sensitive indicators of rangeland condition and can be linked to erosion potential, forage production, wildlife habitat, and risk of invasion by non-native plant species. Vegetation composition, measured in almost all monitoring protocols, is sensitive (when combined with bare ground) to changes in the status of terrestrial ecosystems. Composition is also necessary for determining Fire Regime Condition Class (FRCC). Non-native invasive species have the potential to alter ecological processes and displace native plant communities. Plant species of management concern contribute notably to biotic integrity and biodiversity. Vegetation height is necessary to characterize vegetation structure-an important determinant of wildlife habitat and potential for wind erosion. Proportion of a site with large inter-canopy gaps is strongly related to wind and water erosion rates and can be used in conjunction with composition and height information to describe wildlife habitat.

Two contingent indicators are measured only when there is reason to believe problems exist on the site related to either indicator (Table 1). Soil stability is an indicator of changes in soil erodibility and soil organic matter cycling. This indicator should be evaluated on sites where soils are not expected to be highly stable. Accumulation of toxinsan indicator of major threats to human and environmental health-should be evaluated when there is reason to believe that significant accumulations of toxins exist, such as where a chemical spill has occurred.

\section{Core Methods}

A method is a specific technique or protocol for measuring an indicator. There may be many suitable methods for measuring an indicator. Methods may differ from each other in terms of the type of information recorded (e.g., cover, density, or abundance), precision, repeatability, potential for bias, or cost. Some methods are capable of measuring many indicators. When combining measurements of an indicator made at different times or from different areas, it is necessary that the information be of the same type. However, the 

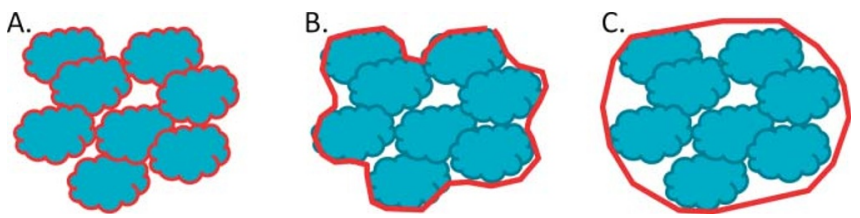

Figure 2. Example of a difference in method that results in incompatible data. Blue shapes represent parts of a single plant (e.g., a sagebrush [Artemisia spp.] plant). When measuring plant cover, the decision of whether to measure foliar or total canopy cover must be made. Foliar cover (A) measures just the exposed plant area, whereas total canopy cover (B) measures the area of influence of the plant. Relative to each other, a total canopy cover method will produce greater estimates of plant cover and lesser estimates of bare ground cover than will a foliar cover method. Because the two methods are measuring different aspects of plant cover, estimates from the two methods cannot be combined. Canopy cover estimates are often biased by differences in how the canopy "margins" are defined (B vs. C) by different observers and by the same observer at different times of the day or for different species or morphologies.

measurements can have differing precision. Often, small differences in the way a method is implemented (i.e., data are collected) can limit the ability to combine data for comparative analysis.

An example of this is the difference between using foliar cover (i.e., actual exposed plant area) versus canopy cover (i.e., perceived area of influence of a plant) when measuring cover indicators (Fig. 2). For the same area, canopy cover measurements will be greater than those for foliar cover. This could have implications when comparing two measurements taken using different definitions, when trying to combine these data to make larger-scale inferences, or when trying to use the data to train remotely sensed imagery. Also, there are many different definitions of canopy cover. ${ }^{7}$ Without consistent methods, combining data sets becomes difficult or impossible. This example illustrates methodological differences that can affect the accuracy of measurements (i.e., the estimates derived from each method would be different). Other types of modifications, such as changing the number of measurements per plot, can often be made without affecting accuracy, though they may affect precision.

The first four core indicators are measured using the line-point intercept (LPI) method for foliar cover ${ }^{8,9}$ along with modifications for data collection in tree cover augmented by a plot-level plant species inventory (Table 2). The fifth core indicator, vegetation height, is measured by recording height of the highest living or dead vegetation within a $15-\mathrm{cm}$ radius of a subset of the LPI points per transect. In many cases, such as the NRI, and for monitoring of sage grouse habitat, the maximum height of both

\section{Table 2. Recommended methods and measurements for core and contingent indicators

\begin{tabular}{|l|l|ll} 
Method & Indicator(s) & Description
\end{tabular}

For core indicators

plot-level species inventory 4

Bare ground

Vegetation composition

Non-native invasive

species

Plant species of management concern

Vegetation height ${ }^{4}$

Vegetation height

Canopy gap intercept ${ }^{4,5}$

Proportion of soil surface in large intercanopy gaps
Line-point intercept ${ }^{4,5}$ with

Line-point intercept (LPI) is a rapid and accurate method for quantifying foliar cover of vegetation and bare ground. However, because LPI can underestimate cover of uncommon species, this method is supplemented with searches of a 150-foot (45.6-m) diameter standard plot for at least 15 minutes and until new species detections are more than 2 minutes apart. When performing LPI within tree-cover, a modified pin method (e.g., a pivotable laser or extendable pin) will be used to capture over-story cover.

Height of tallest leaf or stem of woody and herbaceous vegetation (living or dead) within a 6 -inch $(15-\mathrm{cm})$ radius for points along a transect. If vegetation is $>10$ feet, a standard tape-and-clinometer method should be used to estimate vegetation height.

Canopy gap intercept measures the proportion of a line covered by large gaps between plant canopies and is an important indicator of the potential for erosion. Use 1 -foot $(30-\mathrm{cm})$ minimum gaps.

\section{For contingent indicators}

Soil stability 4,5

Soil aggregate stability

Soil sample collection ${ }^{6}$

\section{Accumulation of} toxins
This test measures the soil's stability when exposed to rapid wetting and provides information on integrity of soil aggregates, degree of structural development, resistance to erosion, and soil biotic integrity.

Presence and concentrations of toxins are assessed through collection of three samples from the soil surface and at one sample at depths of $0-4$ inches $(0-10 \mathrm{~cm})$ and $4-8$ inches $(10-20 \mathrm{~cm})$ using a soil corer and following the Forest Inventory and Analysis protocol. 


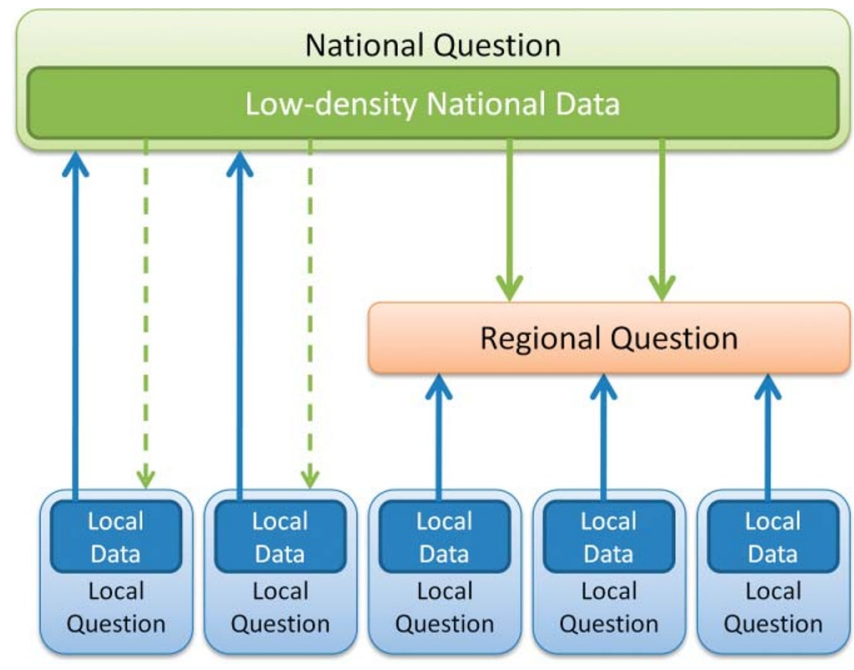

Figure 3. The Assessment, Inventory, and Monitoring (AIM) Strategy approach to sample design is intended to be able to use local and national data to answer monitoring and assessment questions at many different scales. Data collected by Bureau of Land Management (BLM) field and district offices to address local questions (e.g., Has a change in grazing season of use reduced the amount of bare ground cover within an allotment?) can be incorporated into a low-density national sampling grid to improve the accuracy and precision of national indicator estimates. An example of a national-level question might be, "What proportion of all BLM allotments are experiencing increases in cover and distribution of invasive species?" If one of the national sampling grid locations happens to fall within a local monitoring area, it can be directly incorporated into the local sampling (dashed arrows). Both local and national data can be combined to answer regional monitoring and assessment questions such as, "Has energy development on BLM lands increased the prevalence and size of large inter-canopy gaps?"

woody and herbaceous vegetation is recorded. Intercanopy gaps are measured using the canopy-gap intercept method. $^{8}$

Soil stability is measured with the soil aggregate stability method (Table 2). ${ }^{8,9}$ Toxins are measured by taking soil samples at the surface and at two depths following the protocol employed by the Forest Service's Forest Inventory and Analysis program. ${ }^{10}$

\section{Scalable Sample Design}

Both consistent data collection methods and consistent probability-based (i.e., randomized) sample design are required to ensure that data can be integrated and applied to future (and unanticipated) questions. A "scalable sample design" means that samples taken at one scale (e.g., within a single monitoring unit) can be combined with similarly collected data from one or more other locations to say something about a larger area (i.e., "scaling-up" the data; Fig. 3). Three interrelated concepts underpin the AIM Strategy approach to scalable and flexible sample design:

1) Unbiased-This means explicitly defining a study area to which conclusions can be drawn from the data (i.e., an inference space or population of interest) and ensuring that every location within that area or member of the population has some chance of being selected for sampling. Allowing local BLM staff to define their own study areas according to management needs is an important part of achieving the flexibility desired with the AIM Strategy. This can only happen if the local inference space (i.e., study area or population) is explicitly defined and steps are taken to preserve the study area throughout the process of designing and conducting the sampling.

2) Random, probability based-To be used for monitoring multiple objectives at multiple scales, locations for sampling must be randomly selected. This is important for two reasons. First, random locations ensure there is no intentional bias associated with selecting locations to sample. Second, random locations increase the ability to reuse data collected originally for one purpose for other purposes (i.e., re-tasked). Nonrandom samples can only be qualitatively extrapolated to larger landscapes because there is no way to quantify the level of confidence associated with estimates outside the samples themselves. Even if nonrandom locations were representative of conditions at the time and for the purpose they were selected, they may not be representative for other objectives, and changing circumstances may render them not representative in the future.

Every location within the study area must have a known and nonzero chance of being selected for sampling. These selection probabilities do not need to be the same for all locations; however, the probabilities must be known. Variable selection probabilities can be used to focus the sampling effort on areas likely to change, and if they are set using a variable that is correlated with the core indicators (e.g., remote-sensing vegetation index) a greater sampling efficiency can be realized. The probability that a location was included in the sampling is used to weight the observations when results are calculated.

3) Stratified-Dividing up a sampling area into relatively consistent (i.e., homogeneous) units reduces the variability of the measures within each unit and increases sampling efficiency overall (Fig. 4). Additionally, greater efficiency can be achieved by varying the intensity of sampling by stratum based on expectations of change.

This is primarily a bottom-up approach to producing statistically defensible data where local staff can design sampling strategies following general guidelines to meet local needs but also needs across scales. Strata and selection probabilities can be defined according to local spatial data layers, including soil or ecological site map units, ${ }^{3}$ management boundaries, roads, or vegetation indices derived from remotely sensed imagery. Strata based on features that are stable over time are preferred (e.g., soil or ecological site map units). Within these guidelines, local staff can define their study areas (i.e., inference space) and use widely available tools for estimating necessary sample sizes and selecting sample locations.

Collating different local-level sampling efforts to answer coarser-scale questions would be difficult if the coverage of 


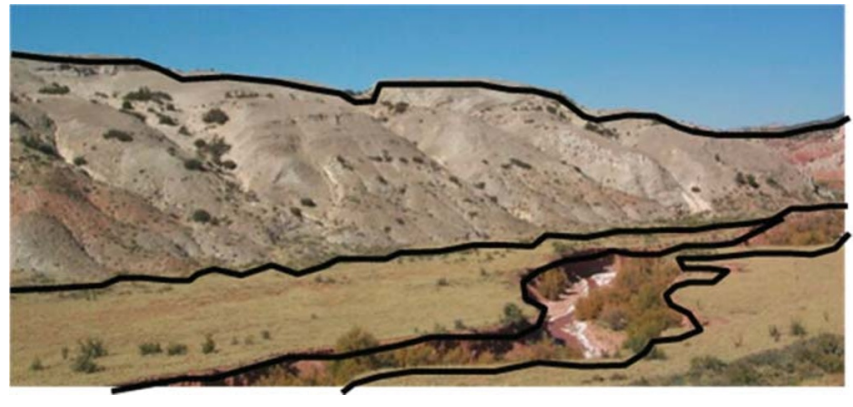

Figure 4. Stratification is the dividing up of a sampling area into relatively homogeneous subunits. Stratification increases sampling efficiency if the variability within each stratum is minimized. Sampling intensity can vary between strata depending on how variable conditions are within the strata or how likely a stratum is to experience change. Figure by J. Van Zee.

structured, local-level sampling was patchy (i.e., if some areas have AIM-based sampling data, but others do not). Combining local-level sampling efforts will be facilitated by the BLM's implementation of the NRI on BLM lands. The NRI provides the ability to draw regional and national inferences about the status and trend of all BLM lands for multiple objectives, and locally derived sampling data sets could be merged into the grid to improve the precision of estimates in those areas (Fig. 3). Aspects of sample design (e.g., stratification or how NRI can be used to inform local monitoring) for the NRI implementation on BLM lands are still being developed.

\section{Remote Sensing Integration}

Remote sensing integration refers to the simultaneous use of field and remote-sensing data for monitoring and assessment. Remote-sensing technologies have much to offer in terms of increasing efficiency, reducing the amount of costly field data that needs to be collected, and improving the ability to monitor large and diverse landscapes. Field data will always be needed to relate measures of reflected radiation to ecosystem indicators (i.e., image training), but they will also be needed to validate remotely sensed data products and to provide information on indicators that cannot be remotely sensed. Remote-sensing integration supported by the AIM Strategy includes the following:

1) Training, validation, or characterization of remotelysensed products-Field data will be used to train and validate remote-sensing-based products like vegetation classifications, landscape-level maps of attributes like bare ground cover, biomass production, and invasive-species prevalence. Additionally, field data are necessary to characterize the plot-level status and trend within landscape patterns (e.g., wildlife corridors) identified via remote sensing.

2) Aiding in selection of field sampling locations-Remotesensing products such as vegetation indices and classifications capture many landscape patterns of interest for management, and these data can be used to help derive
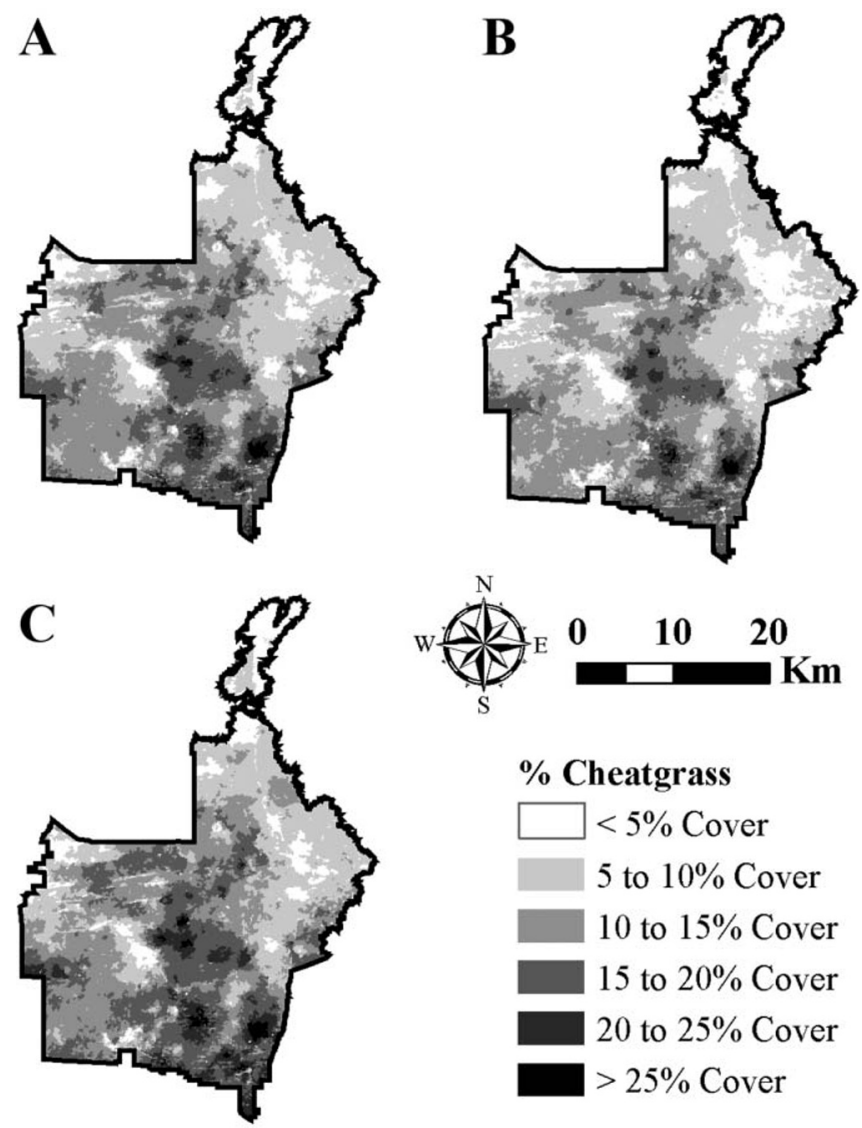

$\%$ Cheatgrass

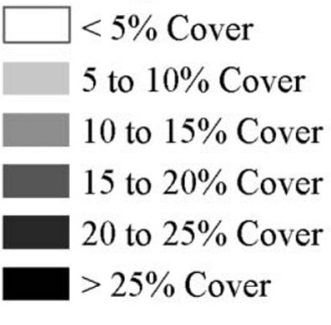

Figure 5. Example of an integrated field and remote-sensing technique that can be used to make spatial predictions of rangeland indicators. In this example, cover of invasive cheatgrass (Bromus tectorum) was predicted for a Bureau of Land Management allotment in southern Idaho using regression kriging from Landsat satellite imagery and field measurements from 346 sample points $(\mathbf{A})$. Also shown are the lower $(\mathbf{B})$ and upper (C) $95 \%$ confidence intervals on the prediction. Adapted from Karl. ${ }^{11}$

strata and sample selection probabilities for field-based monitoring and assessment.

3) Extrapolating field-based data to broader landscapesBeyond training remotely sensed images, field data can be used, via geostatistical and other techniques, to improve the accuracy and precision of remotely sensed estimates of indicator values ${ }^{11}$ (Fig. 5) and to calculate additional indicators that cannot be derived from field data alone, such as measures of habitat patch size or connectivity.

4) Improving field-based estimates with remote-sensing data-The precision of field-based estimates can be significantly improved by adding remote-sensing data as covariates. Statistical techniques like regression estimation $^{12}$ can use the statistical relationship between the field measurements and remote sensing data to improve estimate precision. This could be useful, for instance, in estimating cover of bare ground or species composition in large study areas. This is different from items 1 and 3 above because no spatial prediction is being made, but its advantage is that it increases efficiency, thereby reducing 
the number of field samples needed to detect a given amount of change.

5) Supplement field-based sampling with image-based sampling-Emerging sensor platforms such as those provided by low-flying manned or unmanned aircraft can provide quick and relatively inexpensive mechanism for the collection of very-high-resolution stereo imagery to augment some AIM plot-level data needs. This technique alone cannot address all AIM field data needs outlined above, but it does provide comparable results for a select number of indicators. Data collection need not be limited in distribution due to access issues, or in quantity due to cost and time. Sampling intensities can be greatly increased due to flexibility of the systems.

Integration of remote sensing with field-based data acquisition is a rapidly developing field. The AIM Strategy supports a suite of proven techniques that can be standardized and applied in a consistent way throughout BLM while allowing for the integration of increasingly cost-effective remote sensing tools.

\section{Conclusion}

The ability to combine sets of observations collected at different locations and for different purposes requires both consistent methods and sample designs. By providing this consistency, the above approach creates a flexible monitoring and assessment framework that can be adjusted and supplemented as necessary for local BLM needs while at the same time allowing local and national data sets to be combined to answer wider questions at regional and national scales. Application of the AIM Strategy indicators and methods will help reduce error and increase compatibility among BLM sampling efforts. While generic enough to be accepted by a wide range of users and provide a variety of measures applicable to many different management objectives, the indicators and methods we present here can and should be supplemented by additional indicators to address local needs.

\section{References}

1. Toevs, G. R., J. J. Taylor, C. S. Spurrier, W. C. MacKinnon, AND M. R. Вово. 2011. Bureau of Land Management Assessment, Inventory, and Monitoring Strategy for integrated renewable resources management (draft). Washington, DC, USA: Bureau of Land Management. 27 p.

2. Pyke, D. A., J. E. Herrick, P. Shaver, and M. Pellant. 2002. Rangeland health attributes and indicators for qualitative assessment. Journal of Range Management 55:584-597.

3. KarL, J. W., AND J. E. Herrick. 2010. Monitoring and assessment based on ecological sites. Rangelands 32:60-64.

4. Herrick J. E., S. Wills, J. Karl, and D. Pyke. 2010. Terrestrial indicators and measurements: selection process and recommendations. Available at: http://jornada.nmsu.edu/sites/ default/files/AIM_Terrestrial_Indicators_Selection.pdf. Accessed 7 July 2011.

5. Nusser, S. M., ANd J. J. Goebel. 1997. The National Resources Inventory: a long-term multi-resource monitoring programme. Environmental and Ecological Statistics 4:181-204.
6. Herrick, J. E., V. Lessard, K. E. Spaeth, P. Shaver, R. S. Dayton, D. A. Pyke, L. Jolley, And J. J. Goebel. 2010. National ecosystem assessments supported by scientific and local knowledge. Frontiers of Ecology and the Environment 8:403408.

7. Wilson, J. B. 2011. Cover plus: ways of measuring plant canopies and the terms used for them. Journal of Vegetation Science 22:197-206.

8. Natural Resources Conservation Service. 2009. National resources inventory grazing land on-site study: handbook of instructions. Available at: http://www.nrisurvey.org/nrcs/ range/resource/2009/Instructions/instruction.htm. Accessed 7 July 2011.

9. Herrick, J. E., J. W. Van Zee, K. M. Havstad, L. M. Burkett, AND W. G. Whitford. 2009. Monitoring manual for grassland, shrubland, and savanna ecosystems. Las Cruces, NM, USA: USDA-ARS Jornada Experimental Range. 236 p.

10. US Forest Service. 2007. Forest inventory and analysis national core field guide, version 4.0. Available at: http:// www.fia.fs.fed.us/library/field-guides-methods-proc/. Accessed 7 July 2011.

11. KarL, J. W. 2010. Spatial predictions of cover attributes of rangeland ecosystems using regression kriging and remote sensing. Rangeland Ecology \& Management 63:335-349.

12. Sarndal, E., B. Swensson, and J. Wretman. 2003. Model assisted survey sampling. New York, NY, USA: Springer Verlag. 694 p.

13. Pellant, M., P. Shaver, D. A. Pyke, and J. E. Herrick. 2005. Interpreting indicators of rangeland health, version 4. Denver, CO, USA: US Department of the Interior, Bureau of Land Management, National Science and Technology Center. $123 \mathrm{p}$.

14. MilLER, M. E. 2005. The structure and functioning of dryland ecosystems: conceptual models to inform long-term ecological monitoring. Reston, VA, USA: US Geological Survey. USGS Scientific Investigations Report 2005-5197. 73 p.

Authors are National Assessment, Inventory, and Monitoring Coordinator, Bureau of Land Management, Renerwable Resources and Planning, Washington, DC 20003, USA, GToevs@blm.gov (Toevs); Research Ecologist, USDA Agricultural Research Service, Jornada Experimental Range, Las Cruces, NM 88003, USA (J. Karl); Landscape Ecologist, Bureau of Land Management-National Operations Center, Resource Services, Branch of Assessment and Monitoring, Denver, CO 80225, USA (Taylor); Range Ecologist, Bureau of Land Management, Rangeland Resources, Washington, DC 20003, USA (Spurrier); Range Ecologist, Bureau of Land Management-National Operations Center, Resource Services, Branch of Assessment and Monitoring, Denver, CO 80225, USA (M. Karl); Remote Sensing Specialist, Bureau of Land Management-National Operations Center, Resource Services, Branch of Resource Data, Denver, CO 80225, USA (Bobo); and Research Soil Scientist, USDA Agricultural Research Service, Jornada Experimental Range, Las Cruces, NM 88003, USA (Herrick). 